\title{
Type M Resistance to Macrolides Is Due to a Two-Gene Efflux Transport System of the ATP-Binding Cassette (ABC) Superfamily
}

OPEN ACCESS

Edited by:

Fiona Walsh,

Maynooth University, Ireland

Reviewed by:

Xu Jia,

Chengde Medical College, China

Luca A. Vitali,

University of Camerino, Italy

*Correspondence:

Francesco lannell

francesco.iannelli@unisi.it

Gianni Pozzi

gianni.pozzi@unisi.it

tPresent address:

Marco Cassone,

Division of Geriatric and Palliative Care Medicine, University of Michigan, Ann Arbor, MI, United States

Marco R. Oggioni,

Department of Genetics, University

of Leicester, Leicester,

United Kingdom

Gian Maria Rossolini,

Department of Experimental

and Clinical Medicine, University

of Florence, Florence, Italy

Specialty section:

This article was submitted to Antimicrobials, Resistance

and Chemotherapy,

a section of the journal

Frontiers in Microbiology

Received: 22 February 2018

Accepted: 04 July 2018

Published: 31 July 2018

Citation:

lannelli F, Santoro F, Santagati M, Docquier J-D, Lazzeri E, Pastore G,

Cassone M, Oggioni MR,

Rossolini GM, Stefani S and Pozzi G

(2018) Type M Resistance

to Macrolides Is Due to a Two-Gene

Efflux Transport System of the

ATP-Binding Cassette (ABC)

Superfamily. Front. Microbiol. 9:1670.

doi: 10.3389/fmicb.2018.01670
Francesco lannelli1*, Francesco Santoro ${ }^{1}$, Maria Santagati ${ }^{2}$, Jean-Denis Docquier ${ }^{1}$, Elisa Lazzeri ${ }^{1}$, Gabiria Pastore ${ }^{1}$, Marco Cassone ${ }^{1 \dagger}$, Marco R. Oggioni ${ }^{1 \dagger}$, Gian M. Rossolini't , Stefania Stefani ${ }^{2}$ and Gianni Pozzi ${ }^{1 *}$

'Department of Medical Biotechnologies, University of Siena, Siena, Italy, ${ }^{2}$ Section of Microbiology, Department of Biomedical and Biotechnological Sciences, University of Catania, Catania, Italy

The $\operatorname{mef}(\mathrm{A})$ gene was originally identified as the resistance determinant responsible for type $\mathrm{M}$ resistance to macrolides, a phenotype frequently found in clinical isolates of Streptococcus pneumoniae and Streptococcus pyogenes. MefA was defined as a secondary transporter of the major facilitator superfamily driven by proton-motive force. However, when characterizing the mef(A)-carrying elements Tn1207.1 and $\Phi 1207.3$, another macrolide resistance gene, $m s r(D)$, was found adjacent to mef(A). To define the respective contribution of $\operatorname{mef}(\mathrm{A})$ and $\operatorname{msr}(\mathrm{D})$ to macrolide resistance, three isogenic deletion mutants were constructed by transformation of a $S$. pneumoniae strain carrying $\Phi$ 1207.3: (i) $\Delta m e f(\mathrm{~A})-\Delta m s r(\mathrm{D})$; (ii) $\Delta m e f(\mathrm{~A})-m s r(\mathrm{D})$; and (iii) $m e f(\mathrm{~A})-\Delta m s r(\mathrm{D})$. Susceptibility testing of mutants clearly showed that $m s r(D)$ is required for macrolide resistance, while deletion of $\operatorname{mef}(\mathrm{A})$ produced only a twofold reduction in the minimal inhibitory concentration (MIC) for erythromycin. The contribution of $\mathrm{msr}(\mathrm{D})$ to macrolide resistance was also studied in S. pyogenes, which is the original host of $\Phi 1207.3$. Two isogenic strains of S. pyogenes were constructed: (i) FR156, carrying $\Phi 1207.3$, and (ii) FR155, carrying $\Phi 1207.3 / \Delta m s r(D)$. FR155 was susceptible to erythromycin, whereas FR156 was resistant, with an MIC value of $8 \mu \mathrm{g} / \mathrm{ml}$. Complementation experiments showed that reintroduction of the $m s r(D)$ gene could restore macrolide resistance in $\Delta m s r(\mathrm{D})$ mutants. Radiolabeled erythromycin was retained by strains lacking $m s r(\mathrm{D})$, while $m s r(D)$-carrying strains showed erythromycin efflux. Deletion of mef(A) did not affect erythromycin efflux. This data suggest that type $M$ resistance to macrolides in streptococci is due to an efflux transport system of the ATP-binding cassette (ABC) superfamily, in which mef(A) encodes the transmembrane channel, and $\operatorname{msr}(\mathrm{D})$ the two ATP-binding domains.

Keywords: ABC transporter, macrolide efflux, $\Phi 1207.3$, prophage, $\operatorname{mef}(\mathrm{A}), \operatorname{msr}(\mathrm{D})$, Streptococcus pneumoniae, Streptococcus pyogenes

\section{INTRODUCTION}

Macrolides are antibiotic compounds composed of 14 (erythromycin and clarithromycin), 15 (azithromycin), or 16 (josamycin, spiramycin, tylosin)-membered lactones to which amino and/or neutral sugars are linked (Roberts et al., 1999; Roberts, 2008). Resistance to macrolides in streptococci is usually associated with two major mechanisms: (i) target site modification, arising 
from the presence of erm(B) or erm(A) subclass erm(TR) belonging to the class of erm (erythromycin ribosome methylation) methylase genes (Leclercq and Courvalin, 1991; Seppala et al., 1998) and (ii) drug efflux, associated to the presence of $m e f(\mathrm{~A})$ (macrolide efflux) genes (Clancy et al., 1996; Sutcliffe et al., 1996; Tait-Kamradt et al., 1997). Methylation of $23 \mathrm{~S}$ rRNA causes a reduced binding to macrolide, lincosamide, and streptogramin $\mathrm{B}$ antibiotics $\left(\mathrm{MLS}_{\mathrm{B}}\right.$ phenotype), whereas active efflux of macrolides confers a low level of resistance to resistance only to 14- and 15-membered macrolides, but not to 16-membered macrolides, lincosamides, and streptogramin B antibiotics (M phenotype; Weisblum, 1995; Roberts et al., 1999).

The macrolide efflux mef(A) gene was originally described in Streptococcus pyogenes (Clancy et al., 1996) while the allelic variant mef(E) was first described in Streptococcus pneumoniae (Tait-Kamradt et al., 1997). These variants are highly homologous (about 90\% nucleotide identity) and are grouped in the same mef(A) class of macrolide resistance genes (Roberts et al., 1999). The mef(A) gene was not found only in S. pyogenes and S. pneumoniae, but also in a wide variety of other streptococcal species such as Streptococcus agalactiae, Streptococcus mitis, Streptococcus oralis, and Streptococcus salivarius, in other Gram-positive genera including Corynebacterium, Enterococcus, Micrococcus, and Staphylococcus, and in Gram-negative genera such as Acinetobacter, Bacteroides, and Neisseria (Roberts et al., 1999; Klaassen and Mouton, 2005; Santagati et al., 2009). In some countries, the mef(A) gene has become more common than erm (B) in macrolide-resistant S. pneumoniae and S. pyogenes (Green et al., 2006; Reinert et al., 2006; Rudolph et al., 2013) and it has been used as a marker for molecular epidemiology studies (Daly et al., 2004; Klaassen and Mouton, 2005; Amezaga and McKenzie, 2006). The mef(A) allelic variants are carried by different genetic elements. In $S$. pneumoniae, we described a 7244-bp non-conjugative element named Tn1207.1 carrying $m e f(\mathrm{~A})$, whereas $m e f(\mathrm{E})$ was found to be carried by the 5532bp pneumococcal genetic element (mega; Santagati et al., 2000; Gay and Stephens, 2001; Del Grosso et al., 2002, 2006). In S. pyogenes, mef(A) is carried by $\Phi 1207.3$, a 52,491-bp prophage which we found in the erythromycin-resistant clinical strain $2812 \mathrm{~A}$, transferable to a variety of streptococcal species and whose left 7244 -bp sequence is $100 \%$ identical to pneumococcal Tn1207.1 (Santagati et al., 2003; Pozzi et al., 2004; Iannelli et al., 2014a). Other mef(A)-carrying prophages in clinical isolates of S. pyogenes include $\Phi 10394.4$ (Banks et al., 2003), Фm46.1, and its variant VP_00501.1 (Brenciani et al., 2004, 2010; Vitali et al., 2016).

At first, $\operatorname{Mef}(\mathrm{A})$ was defined as a secondary transporter of the major facilitator superfamily (MFS) ${ }^{1}$ and considered to be the only gene product responsible for type $\mathrm{M}$ macrolide resistance, as shown by cloning and functional characterization in Escherichia coli (Clancy et al., 1996). However, in mef(A)-carrying genetic elements such as Tn1207.1 and $\Phi 1207.3$, another macrolide resistance gene, $m s r(\mathrm{D})$, was always found adjacent to $m e f(\mathrm{~A})$, and $m s r(D)$ was shown to contribute to the macrolide efflux resistance of streptococcal strains carrying the $m e f(\mathrm{~A})-m s r(\mathrm{D})$

${ }^{1}$ http://www.tcdb.org/ tandem pair (Iannelli et al., 2004; Ambrose et al., 2005; Vitali et al., 2016; Zhang et al., 2016; Tatsuno et al., 2018).

To assess the relative contribution of $m e f(A)$ and $m s r(D)$ to macrolide efflux, we generated in-frame isogenic mutants of S. pneumoniae and S. pyogenes carrying $m e f(\mathrm{~A})-m s r(\mathrm{D})$ to be used in functional studies. Mutant strains were tested by (i) determining the minimal inhibitory concentration (MIC) of erythromycin and (ii) assaying the actual intracellular accumulation of radiolabeled erythromycin. Results of functional studies were in accordance with bioinformatics analysis predicting that the tandem $m e f(\mathrm{~A})-m s r(\mathrm{D})$ gene pair encodes an efflux transport system of the ATP-binding cassette (ABC) superfamily.

\section{MATERIALS AND METHODS}

\section{Bioinformatic Softwares}

Protein sequence analysis was performed with the softwares Phyre2 (Kelley et al., 2015) and TMpred. Conserved aminoacids were identified with the PSI-BLAST multiple sequence alignment. Terminator sequence was predicted with RNAstructure ver. 5.02 (Mathews Lab). Nucleotide sequence analysis was performed using Microbial Nucleotide BLAST with the Megablast algorithm ${ }^{2}$.

\section{Bacterial Strains and Growth Conditions}

Streptococcal strains used in this work and their relevant properties are reported in Table 1. Bacteria were routinely grown in Tryptic Soy Broth (TSB) or Tryptic Soy Agar (Difco) supplemented with $3 \%$ horse blood at $37^{\circ} \mathrm{C}$. When required, $500 \mu \mathrm{g} / \mathrm{ml}$ streptomycin, $10 \mu \mathrm{g} / \mathrm{ml}$ novobiocin, $0.5 \mu \mathrm{g} / \mathrm{ml}$ erythromycin, $100 \mu \mathrm{g} / \mathrm{ml}$ spectinomycin, and $3 \mu \mathrm{g} / \mathrm{ml}$ chloramphenicol were added to both liquid and solid media.

\section{Construction of the Isogenic S. pneumoniae Mutant Strains}

To allow construction of mutants deleted for the macrolide resistance genes object of this study, and to facilitate further analysis, it was essential to work in a $S$. pneumoniae strain which is readily transformable and whose genomic sequence is available (Hoskins et al., 2001). For this reason, we transferred $\Phi 1207.3$ to the chromosome of unencapsulated laboratory strains of S. pneumoniae which are derived from type 2 strain D39 (Iannelli et al., 1999; Pearce et al., 2002).

Integration of Tn1207.1 or $\Phi 1207.3$ into the chromosome of $S$. pneumoniae occurs within the cds of $c e l B$, a gene involved in DNA uptake during transformation, and disruption of $c e l B$ leads to impairment of competence for genetic transformation in S. pneumoniae strains carrying Tn1207.1 or $\Phi 1207.3$ (Santagati et al., 2000, 2003). For this reason, strain FR125 was constructed in which celB was deleted and integration of $\Phi 1207.3$ after mating occurred at a different chromosomal location (Iannelli and Pozzi, unpublished results). Subsequently, strain FR183, a

\footnotetext{
${ }^{2}$ https://blast.ncbi.nlm.nih.gov/Blast.cgi?PAGE_TYPE $\sim \sim$ BlastSearch\&BLAST_
} SPEC $\sim \sim$ MicrobialGenomes 
TABLE 1 | Bacterial strains.

\begin{tabular}{|c|c|c|}
\hline Strain & Relevant properties ${ }^{a}$ & Origin (references) \\
\hline \multicolumn{3}{|c|}{ S. pneumoniae } \\
\hline D39 & Type 2 Avery's strain & Pearce et al., 2002; Lanie et al., 2007 \\
\hline $\mathrm{R} \times 1$ & Unencapsulated derivative of D39 & Smith and Guild, 1979; Pearce et al., 2002 \\
\hline DP1004 & Unencapsulated derivative of D39; str-41; $\mathrm{Sm}^{\mathrm{R}}$ & Salles et al., 1992; lannelli and Pozzi, 2004 \\
\hline FP11 & Rx1 unencapsulated competence deficient derivative, $\Delta$ comC; $^{2} \mathrm{Nov}^{R}, \mathrm{Cm}^{\mathrm{R}}$ & lannelli and Pozzi, 2007; Santoro et al., 2010 \\
\hline FR122 & FP11 derivative carrying $\Phi 1207.3 ; \mathrm{Nov}^{\mathrm{R}}, \mathrm{Cm}^{\mathrm{R}}, \mathrm{Em}^{\mathrm{R}}$ & This study \\
\hline FR125 & $\begin{array}{l}\text { Rx1 unencapsulated competence deficient derivative carrying } \Phi \text { 1207.3; } \Delta \text { comC, } \\
\text { str-41, } \Delta \text { celB; } \mathrm{Cm}^{\mathrm{R}}, \mathrm{Sm}^{\mathrm{R}}, \mathrm{Spc}^{\mathrm{R}}, \mathrm{Em}^{\mathrm{R}}\end{array}$ & lannelli and Pozzi, unpublished results \\
\hline FR183 & $\begin{array}{l}\text { DP1004 derivative carrying } \Phi 1207.3 \text { (by transformation with FR125 chromosomal } \\
\text { DNA); } \mathrm{Sm}^{\mathrm{R}}, \mathrm{Em}^{\mathrm{R}}\end{array}$ & This study \\
\hline FP39 & FR183 derivative, $\Delta m e f(\mathrm{~A})$ and $\Delta m s r(\mathrm{D}) ; \mathrm{Sm}^{\mathrm{R}}, \mathrm{Cm}^{\mathrm{R}}$ & This study \\
\hline FP40 & FR183 derivative, $\Delta$ mef(A); $\mathrm{Sm}^{\mathrm{R}}, \mathrm{Em}^{\mathrm{R}}, \mathrm{Cm}^{\mathrm{R}}$ & This study \\
\hline FP59 & FR183 derivative, $\Delta m s r(\mathrm{D}) ; \mathrm{Sm}^{\mathrm{R}}, \mathrm{Spc}^{\mathrm{R}}$ & This study \\
\hline FP92 & $\begin{array}{l}\text { FP59 derivative, } m s r(D) \text { (by transformation with a } m s r(D) \text {-containing PCR fragment } \\
\text { amplified from FR183); } \mathrm{Sm}^{R}, \mathrm{Em}^{\mathrm{R}}\end{array}$ & This study \\
\hline FR139 & FP11 derivative carrying $\Phi 1207.3-\Delta m s r(D)$ (by conjugation with FP59); $\mathrm{Nov}^{R}, \mathrm{Spc}^{\mathrm{R}}$ & This study \\
\hline \multicolumn{3}{|c|}{ S. pyogenes } \\
\hline D471 & Streptomycin-resistant serotype M6 strain from RU collection, $\mathrm{Sm}^{\mathrm{R}}$ & Scott and Fischetti, 1983 \\
\hline FR156 & D471 derivative carrying $\Phi 1207.3$ (by conjugation with FR122); $\mathrm{Sm}^{\mathrm{R}}, \mathrm{Em}^{\mathrm{R}}$ & This study \\
\hline FR155 & D471 derivative carrying $\Phi 1207.3-\Delta m s r(\mathrm{D})$ (by conjugation with FR139); $\mathrm{Sm}^{\mathrm{R}}, \mathrm{Spc}^{\mathrm{R}}$ & This study \\
\hline FR160 & FR155 derivative carrying $\Phi 1207.3$ (by conjugation with FR122); $\mathrm{Sm}^{\mathrm{R}}, \mathrm{Em}^{\mathrm{R}}, \mathrm{Spc}^{\mathrm{R}}$ & This study \\
\hline
\end{tabular}

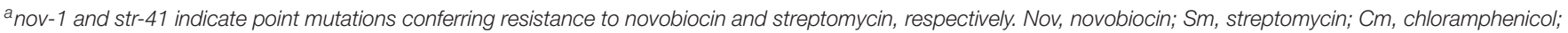
Tc, tetracycline; Em, erythromycin; Spc, spectinomycin; Km, kanamycin.

transformable S. pneumoniae DP1004 derivative strain carrying $\Phi 1207.3$ integrated elsewhere than $c e l B$, was obtained by transformation with the chromosomal DNA purified from FR125 (Table 1).

\section{Matings}

Mating experiments were performed as already described (Iannelli and Pozzi, 2007). Briefly, cells were grown separately in presence of appropriate antibiotics until the end of exponential phase $\left(\mathrm{OD}_{590 \mathrm{~nm}}=0.8\right)$. Cells were mixed at $1: 10$ ratio (Donor/Recipient), harvested by centrifugation and plated. Following 4-h incubation, cells were harvested by scraping the plates. Selection of recombinants was carried out with a multilayer plating procedure. Transfer frequencies were determined by plating alone each parent strain that was also checked for spontaneous antibiotic resistance acquisition.

\section{Oligonucleotide Primers}

Oligonucleotide primers used for mutagenesis, sequencing, and PCR selection of recombinant strains are listed in Supplementary Table S1.

\section{PCR Mutagenesis}

Gene splicing by overlap extension (gene SOEing) was used to generate the mutagenic constructs for gene deletions as already described (Pearce et al., 2002; Iannelli and Pozzi, 2004). The deletion of $m e f(\mathrm{~A})$ and $m s r(\mathrm{D})$ genes was obtained with a mutagenic construct containing the ami/cat cassette flanked at the left by a 195-bp DNA fragment corresponding to nucleotides $3112-3307$ of $\Phi 1207.3$ and at the right by a fragment corresponding to the 532 nucleotides located downstream of the $m s r(\mathrm{D})$ stop codon. The ami/cat chloramphenicol-resistance cassette was obtained with the primer pair IF38/IF39 using the E. coli pEVP3 plasmid as template (Claverys et al., 1995). The flanking regions were amplified, respectively, with the primer pairs IF105/F35 and IF183/IF175 from chromosomal DNA of FR183. To minimize possible polar effects, the mef(A) coding sequence (cds) was deleted in-frame by allelic replacement with the cds of the cat chloramphenicol-resistance gene. The cat cds was amplified with primer pair IF184/IF39 from a $\Omega$ cat(pC194)carrying strain (Iannelli et al., 2014b) and flanked by a 1116bp DNA region located upstream mef(A) start codon and a DNA fragment spanning the 449 nucleotides downstream of the $m e f(\mathrm{~A})$ stop codon, obtained, respectively, with the primer pairs IF176/IF182 and IF185/F20 from FR183 chromosome. A genetic construct containing the ami/spc cassette flanked by the 281-bp and 532-bp DNA fragments located respectively upstream and downstream of $m s r(\mathrm{D}) \mathrm{cds}$ was produced to inactivate $m s r(D)$. The primer couple IF100/IF101 was used to amplify the spectinomycin-resistance cassette from the pR412 plasmid (Martin et al., 2000). This cassette was flanked by the $m s r(\mathrm{D})$ flanking segments amplified with the primer pair IF171/IF174 from FR183 chromosome. Linear PCR constructs were used directly as donor DNA in transformation experiments. Mutant strains were selected for acquisition of chloramphenicol or spectinomycin resistance and the desired mutations were confirmed by sequencing. PCR-based selection of S. pneumoniae complemented strains was carried out with the primers pair IF169/IF170 according to the protocol already described (Iannelli and Pozzi, 2004). 


\section{Erythromycin Sensitivity Determination}

The MIC was assessed by microdilution techniques as suggested by the (Clinical and Laboratory Standards Institute [CLSI], 2017). Briefly, MICs were determined as follows: bacterial cells were grown until exponential growth phase $\left(\mathrm{OD}_{590 \mathrm{~nm}}=0.25\right)$ in TSB medium and stored at $-70^{\circ} \mathrm{C}$ in $10 \%$ glycerol, then frozen cultures were thawed and diluted at $5 \times 10^{4} \mathrm{CFU} / \mathrm{ml}$ in TSB broth containing serial twofold dilutions of antibiotic, and incubated at $37^{\circ} \mathrm{C}$ for $18 \mathrm{~h}$. Bacterial growth was determined turbimetrically using the microplate ELISA reader VERSAmax (Molecular Devices). Results are presented as the geometric mean and are derived from at least three experiments. S. pneumoniae ATCC49619 was used as reference control strain as recommended (Clinical and Laboratory Standards Institute [CLSI], 2017).

\section{Erythromycin Efflux Assay}

Frozen starter culture was diluted 100 -fold in $100 \mathrm{ml}$ warm TSB medium and grown at $37^{\circ} \mathrm{C}$ until an optical density at $590 \mathrm{~nm}$ of 0.25 . Radiolabeled erythromycin $\left[\mathrm{N}\right.$-methyl $\left.-{ }^{14} \mathrm{C}\right]$ $(50 \mu \mathrm{Ci} / \mathrm{mmol}$, purchased from Bio Trend, Germany) was added at a final concentration of $0.2 \mu \mathrm{g} / \mathrm{ml}$ (Sutcliffe et al., 1996). Erythromycin uptake was assessed by taking $5-\mathrm{ml}$ samples from each culture every $10 \mathrm{~min}$ for $40 \mathrm{~min}$ after the addition of $\left[{ }^{14} \mathrm{C}\right]$ erythromycin, filtering samples with prewet GF/C glass microfiber filters (Whatman), and washing the filters twice with $5 \mathrm{ml}$ of a $0.9 \% \mathrm{NaCl}$ and $1 \mu \mathrm{g} / \mathrm{ml}$ erythromycin solution (Sutcliffe et al., 1996). Filters were dried O.N., dissolved in $10 \mathrm{ml}$ of Insta-Gel Plus liquid (Packard) and the $\left[{ }^{14} \mathrm{C}\right]$ erythromycin cell-associated was counted with a TRI-CARB 2000 CA Liquid Scintillation Analyzer (Packard).

\section{RESULTS}

\section{$m e f(\mathrm{~A})$ and $m s r(\mathrm{D})$ Encode an $\mathrm{ABC}$ Transport System}

In clinical isolates of macrolide resistant streptococci, the macrolide efflux gene, mef(A), is found in tandem with $m s r(\mathrm{D})$, a gene which encodes for an ATP-binding transporter (Santagati et al., 2000, 2003; Gay and Stephens, 2001; Banks et al., 2003; Brenciani et al., 2004). Bioinformatic analysis of the prototypic tandem mef(A)-msr(D) from Tn1207.1 (Santagati et al., 2000) and their flanking regions revealed that they constitute a two-gene operon which is $3290 \mathrm{bp}$ long, with only one putative promoter sequence -35 TTGCTT, extended -10 TGTGTTAAAAT (nucleotides 2890-2895 and 2908-2918, respectively, Genbank AF227520) located upstream of the mef(A) start codon and a single predicted terminator sequence (nucleotides 6143-6179, Genbank AF227520) located downstream of the $m s r(\mathrm{D})$ stop codon. As previously demonstrated (Ambrose et al., 2005; Chancey et al., 2015), RT-qPCR analysis confirmed that $m e f(\mathrm{~A})$ and $m s r(\mathrm{D})$ genes were part of a single transcriptional unit (data not shown). Sequence analysis of the $\operatorname{Mef}(A)$ protein with TMpred showed the presence of 12 predicted transmembrane domains, which have the potential to form six transmembrane helices (Figure 1). When the protein sequence of $\operatorname{Msr}(\mathrm{D})$ was analyzed by the PSI-BLAST multiple sequence alignment, it was possible to show the presence of two nucleotide binding domains (NBDs) typical of ATP-binding transporters (spanning amino acids 12-154, and 304-463, Genbank AAG12999.1), and a long predicted alpha helical structure (spanning amino acids 181227) between the two NBDs (Figure 1). Altogether, mef(A) and $m s r(\mathrm{D})$ appear to constitute a two-gene efflux transport system of the $\mathrm{ABC}$ superfamily, mef(A) encodes the pore (transmembrane channel), and $m s r(\mathrm{D})$ the two ATP-binding domains.

\section{$m e f(A)$ and $\operatorname{msr}(\mathrm{D})$ Are Found in Tandem in Bacterial Genomes}

The mef(A) gene sequence (GenBank AF227520) was used as a query to interrogate the database of 20,187 complete Microbial genomes (accessed in May 2018). The mef(A) gene was found in 37 genomes, of those: 29 were streptococcal genomes and eight belonged to other genera (namely Turicibacter, Clostridium, Enterococcus, Clostridioides, Gardnerella, and Bacillus). In 33 out of 37 cases, the mef(A) gene was in tandem with $m s r(\mathrm{D})$, while in the remaining four cases, $m s r(\mathrm{D})$ was substituted by a gene coding for a putative nucleotidyltransferase. No information on macrolide resistance was available for these four genome strains.

\section{Type M Macrolide Resistance Depends on the ATP-Binding Transporter Msr(D)}

To define the respective contribution of $m e f(\mathrm{~A})$ and $m s r(\mathrm{D})$ gene products to macrolide efflux resistance, isogenic deletion

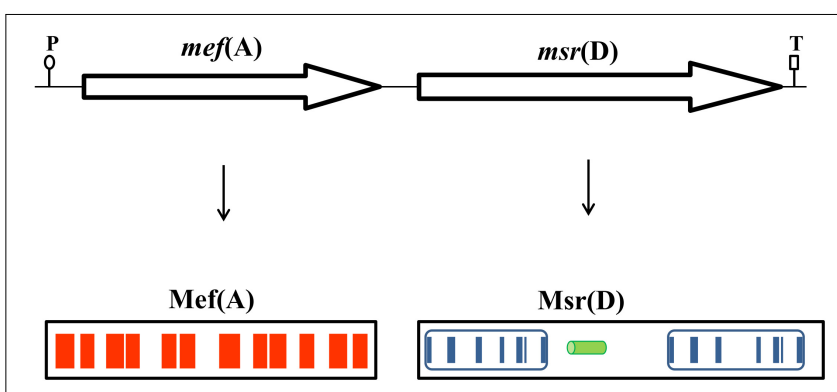

FIGURE 1 | The $\operatorname{mef}(\mathrm{A})-m s r(\mathrm{D})$ operon is 3290-bp-long and includes a single promoter sequence $(P)$ and a terminator sequence $(T)$. $\operatorname{Mef}(A)$ contains 12 transmembrane domains spanning the whole protein (red bars). Msr(D) contains two nucleotide binding domains (NBDs, blue boxes) connected by an alpha helical structure (green cylinder). Each NBD has seven motifs (blue bars): (1) the "A-loop" that provides an aromatic side chain residue that interacts with the adenine ring of bound nucleotide (phenylalanine in both NBDs); (2) the "Q-loop" involved in the interaction with the transmembrane domain and with the $\gamma$-phosphate through a water bond; (3) the "P-loop" (or "Walker-A" motif) that binds the nucleotide; (4) the "LSGGQ motif" (also called "C-loop" or "ABC signature motif") which contacts the nucleotide in the ATP-bound state; (5) the "Walker-B" motif that has a conserved glutamate residue that orchestrates the nucleophilic attack on ATP via a water molecule; (6) the "D-loop" involved in the contact between the two NBDs through an interaction with the Walker-A asparagine residue; and (7) the "switch motif" that contains a histidine side chain thought to contribute to the catalytic reaction. Both C-loop motifs of Msr(D), MSGGE and LSGGE, are not identical to the consensus (LSGGQ). 
mutants were constructed in S. pneumoniae for the mef(A)$m s r(\mathrm{D})$ tandem pair carried by two different genetic elements (Figure 2). Three isogenic deletion mutants were constructed in the $\Phi 1207.3$-carrying strain FR183: (i) FP39, $\Delta m e f(\mathrm{~A})-$ $\Delta m s r(\mathrm{D})$; (ii) FP40, $\Delta m e f(\mathrm{~A})$; and (iii) FP59, $\Delta m s r(\mathrm{D})$. In FP39, a 2748-bp DNA fragment (position 3308-6055, GenBank AY657002) containing $m e f(\mathrm{~A})$ and $m s r(\mathrm{D})$ was deleted and replaced with the 850-bp ami/cat cassette. In FP40, a 1218bp DNA fragment (position 3255-4472, GenBank AY657002) containing the cds of $m e f(A)$ was deleted and replaced in-frame with the 651-bp cds of the cat gene. In FP59, a 1464-bp DNA fragment (position 4592-6055, GenBank AY657002) containing the $m s r(D)$ cds was deleted and replaced with the 894-bp ami/spc cassette (Figure 2 and Table 1).

Sensitivity to erythromycin of the S. pneumoniae strains carrying $m e f(\mathrm{~A})-m s r(\mathrm{D})$ and of their isogenic mutants was tested in liquid medium. It was clearly shown that $m s r(\mathrm{D})$ is required for macrolide resistance (Figure 1 and Table 2). Deletion of mef(A) produced only a twofold reduction of the MIC, while deletion of $m s r(D)$ produced a 64 -fold MIC decrease (8 to $0.12 \mu \mathrm{g} / \mathrm{ml}$ ), and when both genes were deleted, the MIC decreased 128-folds (Table 2). Identical results were also obtained using the allelic
TABLE 2 | Sensitivity to erythromycin.

\begin{tabular}{llcl}
\hline Strain & Genotype & MIC of erytromycin $(\boldsymbol{\mu g} / \mathbf{m l})^{\mathbf{a}}$ & Phenotype \\
\hline \multicolumn{2}{l}{ Streptococcus pneumoniae } & & \\
FR183 & mef(A), $m s r(\mathrm{D})$ & 8 & Resistant \\
FP39 & $\Delta$ mef(A), $\Delta m s r(\mathrm{D})$ & 0.06 & Sensitive \\
FP40 & $\Delta$ mef(A), msr(D) & 4 & Resistant \\
FP59 & mef(A), $\Delta m s r(\mathrm{D})$ & 0.12 & Sensitive \\
DP1004 & - & 0.03 & Sensitive \\
Streptococcus pyogenes & & \\
FR156 & mef(A), msr(D) & 8 & Resistant \\
FR155 & mef(A), $\Delta m s r(\mathrm{D})$ & 0.12 & Sensitive \\
D471 b & - & 0.03 & Sensitive
\end{tabular}

${ }^{a}$ MIC interpretative standard: sensitive $\leq 0.25 \mu \mathrm{g} / \mathrm{ml}$, intermediate $=0.5 \mu \mathrm{g} / \mathrm{ml}$, and resistant $\geq 1 \mathrm{\mu g} / \mathrm{ml}$.

${ }^{b}$ DP1004 and D471 are parental erythromycin sensitive strains used as controls.

variants of $m e f(\mathrm{~A})$ and $m s r(\mathrm{D})$ originally found in a macrolideresistant clinical strain of $S$. pneumoniae formerly described as a "mef(E)-positive isolate" (GenBank AF376746) (Del Grosso et al., 2002, 2004). Deletion mutants were constructed and tested for erythromycin resistance. Results on MIC reduction were identical

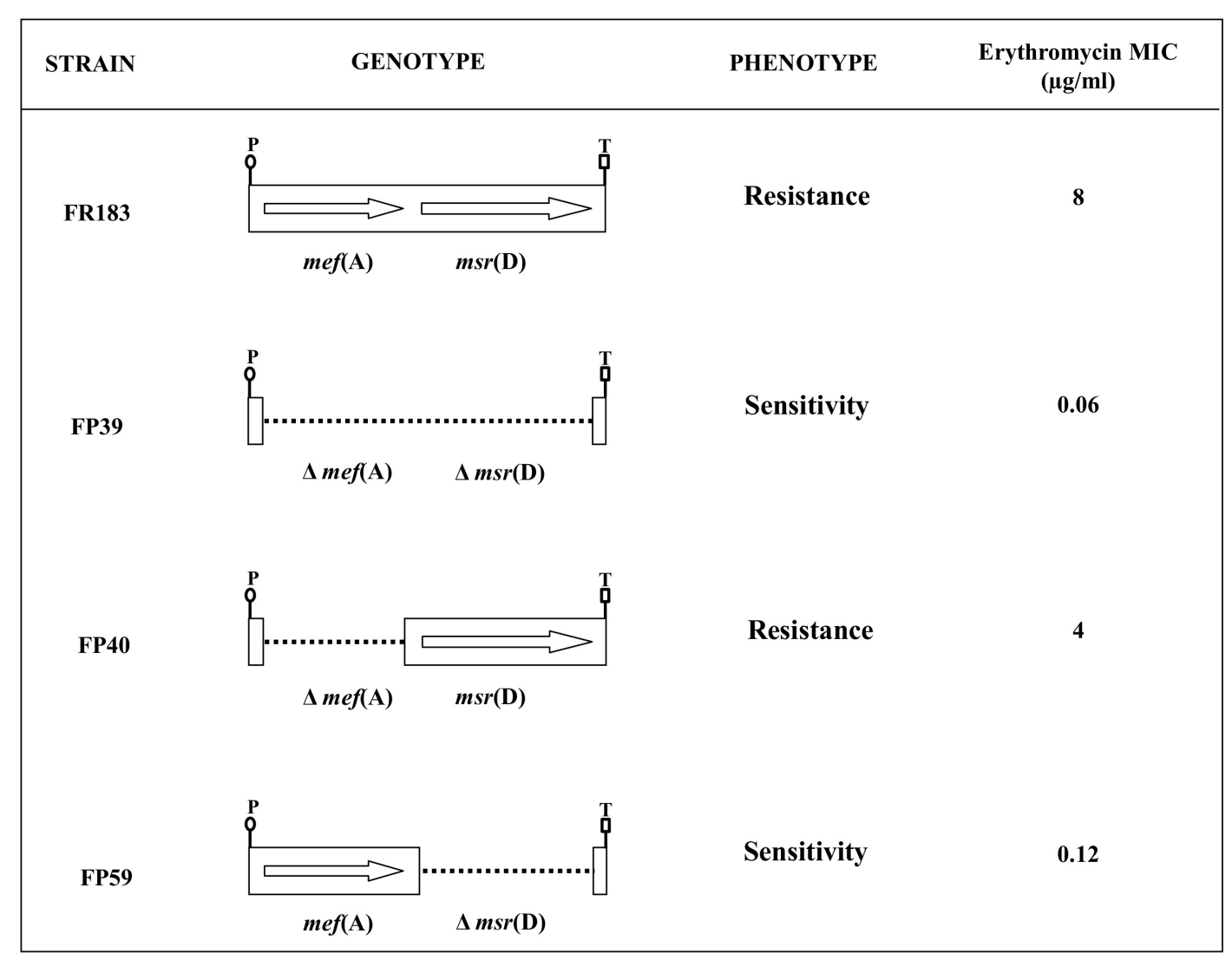

FIGURE 2 | The mef(A)-msr(D) operon, carried by $\Phi 1207.3$, confers type M resistance to macrolides. Isogenic mutants were produced in a $\Phi 1207.3-c a r r y i n g$ S. pneumoniae strain. CDSs were deleted by allelic replacement with mutagenic antibiotic resistance cassettes. In order to minimize polar effect, mef(A) CDS was in frame deleted with the chloramphenicol resistance cat CDS. The genotype and relative erythromycin resistance phenotype of the pneumococcal isogenic strains are schematized. The genes are reported as arrows while dotted lines indicate gene deletions; the transcriptional promoter $(\mathrm{P})$ and the putative transcriptional terminator (T) of the operon are reported. 
to those observed for the $m e f(\mathrm{~A})-m s r(\mathrm{D})$ alleles described above (data not shown).

\section{Complementation of the $\boldsymbol{m s r}(\mathrm{D})$ Deletion}

To confirm the role of $m s r(\mathrm{D})$ in determining type $\mathrm{M}$ resistance to macrolides, deletion of $m s r(\mathrm{D})$ was complemented in S. pneumoniae FP59 carrying $\Phi 1207.3 / \Delta m s r(D)$. Competent cells of FP59 were transformed with a 2206-bp PCR fragment containing $m s r(\mathrm{D})$ obtained from wild type $\Phi 1207.3$, using primer pair IF171/IF175. Transformants were selected by PCR for the presence of the $m s r(\mathrm{D})$ gene, as previously described (Iannelli and Pozzi, 2004). A total of four transformants were selected out of 186 tested. All four showed acquisition of erythromycin resistance, with an MIC value of $8 \mu \mathrm{g} / \mathrm{ml}$. A representative transformant was named FP92, checked by DNA sequencing and used as a control in further experiments.

\section{The $\operatorname{msr}(\mathrm{D})$ Gene in Streptococcus pyogenes}

The contribution of $m s r(\mathrm{D})$ to macrolide resistance was also studied in S. pyogenes, which is the original host of $\Phi 1207.3$ (Santagati et al., 2003). Two isogenic strains of S. pyogenes were constructed: (i) FR156, carrying $\Phi 1207.3$, and (ii) FR155, carrying $\Phi 1207.3 / \Delta m s r(D)$ (Table 1). $\Phi 1207.3$ transfer from $S$. pneumoniae donors to $S$. pyogenes recipients occurred at frequencies of $10^{-7}$ recombinants per donor. FR155 lacking $m s r(D)$ was susceptible to erythromycin (MIC of $0.12 \mu \mathrm{g} / \mathrm{ml}$ ), whereas FR156 was resistant, with an MIC of $8 \mu \mathrm{g} / \mathrm{ml}$ (Table 2). These data show that in S. pyogenes the presence of the mef $(\mathrm{A})$ gene alone determines a fourfold increase of the MIC for erythromycin, while when both $m e f(\mathrm{~A})$ and $m s r(\mathrm{D})$ are present, the MIC is increased by 256 -folds (Table 2).

The $\Phi 1207.3 / \Delta m s r(D)$ S. pyogenes FR155 was complemented in trans transferring a wild type $\Phi 1207.3$ element from FR122. Recombinants were selected for erythromycin resistance acquisition and in a representative recombinant, FR160, the presence of both the wild type and mutated form of $\Phi 1207.3$ was confirmed by DNA sequencing. FR160 showed an erythromycin MIC value of $8 \mu \mathrm{g} / \mathrm{ml}$.

\section{Erythromycin Efflux}

The function of $m e f(\mathrm{~A})$ and $m s r(\mathrm{D})$ was studied by testing incorporation of radiolabeled erythromycin in the deletion mutants (Figure 3). Radiolabeled erythromycin was retained/incorporated by strains lacking $m s r(D)$, while $m s r(\mathrm{D})$-carrying strains showed erythromycin efflux. This was observed both in S. pneumoniae (Figure 3A) and in S. pyogenes (Figure 3B). Accumulation of radiolabeled erythromycin was maximum in the $\Delta m e f(\mathrm{~A})-\Delta m s r(\mathrm{D}) S$. pneumoniae mutant. The deletion of mef(A) did not affect erythromycin efflux: $\Delta m e f(\mathrm{~A})$ mutant showed an erythromycin accumulation comparable to that of the wild type strain, even if the kinetics of accumulation were different (Figure 3A). Both the S. pneumoniae and the $S$. pyogenes $\Delta m s r(\mathrm{D})$ mutants showed an intracellular accumulation of erythromycin which peaked already $10 \mathrm{~min}$ after erythromycin addition (Figures 3A,B). These results were confirmed in a series of control efflux assays which included (i) testing deletion mutants derived a " $m e f(\mathrm{E})$-positive strain" carrying mef(A)-msr(D) allelic variants and (ii) testing complemented strain FP92 (data not shown). Taken together,
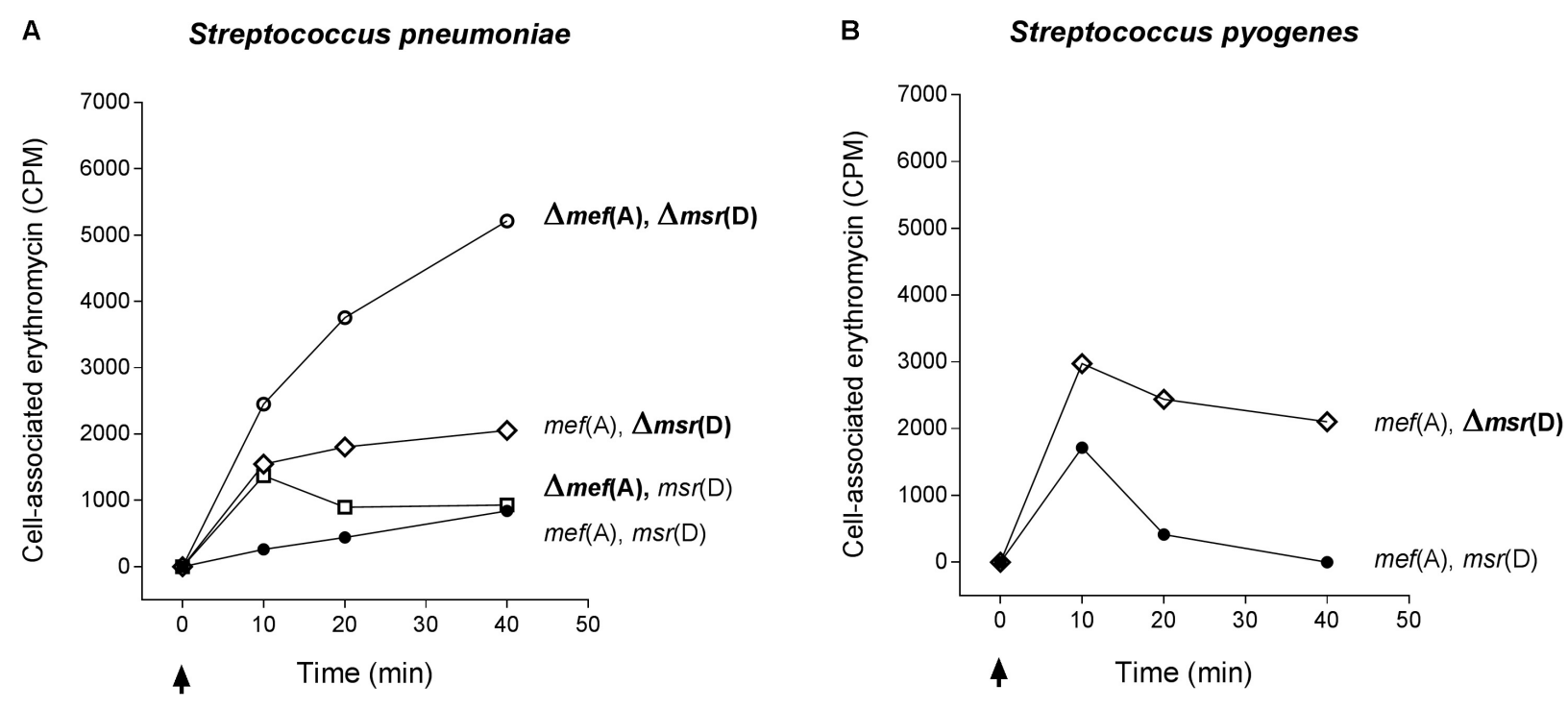

FIGURE 3 | Efflux assay in S. pneumoniae (A) and S. pyogenes (B) isogenic mutant strains. The $\Delta m e f(A)-\Delta m s r(D) S$. pneumoniae mutant showed the maximum accumulation of radiolabeled erythromycin. Erythromycin efflux was not affected by the deletion of mef(A). S. pneumoniae and $S$. pyogenes $\Delta m s r(D)$ mutants showed an intracellular accumulation of erythromycin peaking 10 min after erythromycin addition. Genotype is indicated on the right of each graph. Cell-associated erythromycin is reported as counts per minute (CPM). Arrows indicate the time point at which radiolabeled erythromycin was added. Full circles indicate the wild type strain carrying $m e f(\mathrm{~A})$ and $m s r(\mathrm{D})$, open circles the mef(A) and $m s r(\mathrm{D})$ deletion mutant, open diamonds the $m s r(\mathrm{D})$ deletion mutant, and open squares the mef(A) deletion mutant. In all curves, the CPM baseline value at time 0 was subtracted from the CPM values of each time point. 
these results suggest that type $M$ resistance to macrolides in streptococci is due to an efflux transport system of the $\mathrm{ABC}$ superfamily, in which mef(A) encodes the transmembrane channel, and $m s r(\mathrm{D})$ the two ATP-binding domains.

\section{DISCUSSION}

In this work, protein sequence analysis showed that $\operatorname{Mef}(\mathrm{A})$ contains 12 transmembrane segments and $\operatorname{Msr}(\mathrm{D})$ contains two ATP-binding domains (ABC domains). Analysis of bacterial genomes showed that $m e f(\mathrm{~A})$ and $m s r(\mathrm{D})$ are found in tandem in 33 out of 37 cases. We constructed isogenic deletion mutants in $S$. pneumoniae and $S$. pyogenes to define the respective contribution of $m e f(\mathrm{~A})$ and $m s r(\mathrm{D})$ to macrolide resistance. Physiological studies by MIC determinations and efflux assays, carried out in the original streptococcal hosts, showed that $m s r(D)$ is essential for erythromycin resistance and drug efflux, whereas mef(A) deletion produces a twofold reduction of MIC and does not affect efflux. Thus, it appears that $m e f(\mathrm{~A})$ and $m s r(\mathrm{D})$ encode an $\mathrm{ABC}$ transporter involved in macrolide efflux with $\operatorname{Mef}(A)$ as the transmembrane channel, and $\operatorname{Msr}(\mathrm{D})$ as the cytoplasmatic ATP-binding protein. ABC transporters are multidomain membrane proteins minimally composed of two transmembrane domains and two ATP-binding domains. Transmembrane domains are responsible for binding and transport, while ATPbinding domains for coupling the energy of ATP hydrolysis to conformational changes in the transmembrane domains. These four domains may belong to a single protein or to different proteins.

In E. coli, the majority of transporters are secondary transporters and rely on proton-motive force as a source of energy $^{3}$. Streptococci are fermentative organisms that use ATP as primary source of energy; accordingly, the majority of transporters are ATP-dependent. Because of these metabolic differences, it is likely that $\operatorname{Mef}(\mathrm{A})$ works as a major facilitator when expressed in E. coli (Clancy et al., 1996), while in the original streptococcal host, $\operatorname{Mef}(\mathrm{A})$ is coupled to the cognate ATP-binding protein Msr(D) to function as an efflux system.

In this work, we show that the $m s r(\mathrm{D})$ gene (i) is always in tandem with mef(A) (Figure 1), constituting a single transcriptional unit, and (ii) confers type $\mathrm{M}$ resistance to

${ }^{3}$ http://www.membranetransport.org/transportDB2

\section{REFERENCES}

Ambrose, K. D., Nisbet, A. D., and Stephens, D. S. (2005). Macrolide efflux in Streptococcus pneumoniae is mediated by a dual efflux pump (mel and mef) and is erythromycin inducible. Antimicrob. Agents Chemother. 49, 4203-4209. doi: 10.1128/AAC.49.10.4203-4209.2005

Amezaga, M. R., and McKenzie, H. (2006). Molecular epidemiology of macrolide resistance in beta-haemolytic streptococci of lancefield groups A, B, C and $G$ and evidence for a new mef element in group $G$ streptococci that carries allelic variants of mef and $\operatorname{msr}(\mathrm{D})$. J. Antimicrob. Chemother. 57, 443-449. doi: $10.1093 /$ jac/dki490

Banks, D. J., Porcella, S. F., Barbian, K. D., Martin, J. M., and Musser, J. M. (2003). Structure and distribution of an unusual chimeric genetic element encoding macrolides by an ATP-dependent efflux mechanism. The $m s r(\mathrm{D})$ gene product, $\mathrm{Msr}(\mathrm{D})$, is phylogenetically classified in the ABC-F family of $\mathrm{ABC}$ transporters together with other ATP-binding proteins, such as Vga and Lsa, which confer resistance also to other ribosome targeting antibiotics such as lincosamides, streptogramins, pleuromutilins, and ketolides. Proteins in this family share a general architecture with two NBDs and a long, mostly alpha helical, linker sequence connecting them. The spectrum of antibacterial resistances conferred is variable among members of this family, with some proteins giving resistance to multiple classes of antibiotics (Lenart et al., 2015; Sharkey et al., 2016).

Since deletion of $m e f(A)$ did not affect erythromycin efflux and produced only a twofold reduction on erythromycin $\mathrm{MIC}$, it is possible that in the absence of $\operatorname{Mef}(\mathrm{A}), \operatorname{Msr}(\mathrm{D})$ uses an alternative transmembrane protein to pump the antibiotic out of the cell. A BlastP search in the genomes of $S$. pneumoniae TIGR4 and $S$. pyogenes M1 revealed that there are, respectively, three and one hypothetical transmembrane proteins homologous to $\operatorname{Mef}(\mathrm{A})$. Site-specific mutagenesis of the $m e f(\mathrm{~A})$ homologous genes will be a first step for the identification of alternative Msr(D) cognate transmembrane domains which could complement the $\operatorname{Mef}(\mathrm{A})$ function.

\section{AUTHOR CONTRIBUTIONS}

FI and GPo designed the experiments. FI, FS, MS, J-DD, EL, GPa, and MC performed the experimental work. FI, FS, MO, GR, SS, and GPo analyzed and interpreted the data. FI, FS, and GPo wrote the paper.

\section{FUNDING}

This study was supported by the European Commission grants ANTIRESDEV HEALTH-F3-2009-241446 and Italian Ministry of University and Research, project PRIN 2012 (2012WJSX8K).

\section{SUPPLEMENTARY MATERIAL}

The Supplementary Material for this article can be found online at: https://www.frontiersin.org/articles/10.3389/fmicb. 2018.01670/full\#supplementary-material

macrolide resistance in phenotypically diverse clones of group A Streptococcus. J. Infect. Dis. 188, 1898-1908. doi: 10.1086/379897

Brenciani, A., Bacciaglia, A., Vignaroli, C., Pugnaloni, A., Varaldo, P. E., and Giovanetti, E. (2010). Fm46.1, the main Streptococcus pyogenes element carrying mef(A) and tet(O) genes. Antimicrob. Agents Chemother. 54, 221-229. doi: 10.1128/AAC.00499-09

Brenciani, A., Ojo, K. K., Monachetti, A., Menzo, S., Roberts, M. C., Varaldo, P. E., et al. (2004). Distribution and molecular analysis of mef(A)-containing elements in tetracycline-susceptible and -resistant Streptococcus pyogenes clinical isolates with efflux-mediated erythromycin resistance. J. Antimicrob. Chemother. 54, 991-998. doi: 10.1093/jac/dkh481

Chancey, S. T., Bai, X., Kumar, N., Drabek, E. F., Daugherty, S. C., Colon, T., et al. (2015). Transcriptional attenuation controls macrolide inducible efflux 
and resistance in Streptococcus pneumoniae and in other gram-positive bacteria containing mef/mel(msr(D)) elements. PLoS One 10:e0116254. doi: 10.1371/ journal.pone.0116254

Clancy, J., Petitpas, J., Dib-Hajj, F., Yuan, W., Cronan, M., and Kamath, A. V., et al. (1996). Molecular cloning and fuctional analysis of a novel macrolide-resistance determinant, mefA, from Streptococcus pyogenes. Mol. Microbiol. 22, 867-879. doi: 10.1046/j.1365-2958.1996.01521.x

Claverys, J. P., Dintilhac, A., Pestova, E. V., Martin, B., and Morrison, D. A. (1995). Construction and evaluation of new drug-resistance cassettes for gene disruption mutagenesis in Streptococcus pneumoniae, using an ami test platform. Gene 164, 123-128. doi: 10.1016/0378-1119(95) 00485-O

Clinical and Laboratory Standards Institute [CLSI] (2017). Performance Standards for Antimicrobial Susceptibility Testing, 27th Edn, Wayne, PA: CLSI supplement Clinical and Laboratory Standards Institute.

Daly, M. M., Doktor, S., Flamm, R., and Shortridge, D. (2004). Characterization and prevalence of mefA, mefE, and the associated mrs(D) gene in Streptococcus pneumoniae clinical isolates. J. Clin. Microbiol. 42, 3570-3574. doi: 10.1128/ JCM.42.8.3570-3574.2004

Del Grosso, M., Camilli, R., Iannelli, F., Pozzi, G., and Pantosti, A. (2006). The mef(E)-carrying genetic element (mega) of Streptococcus pneumoniae: insertion sites and association with other genetic elements. Antimicrob. Agents Chemother. 50, 3361-3366. doi: 10.1128/AAC.00277-06

Del Grosso, M., Iannelli, F., Messina, C., Santagati, M., Petrosillo, N., and Stefani, S. et al. (2002). Macrolide efflux genes mef(A) and mef(E) are carried by different genetic elements in Streptococcus pneumoniae. J. Clin. Microbiol. 50, 1230-1240. doi: 10.1128/JCM.40.3.774-778.2002

Del Grosso, M., Scotto d'Abusco, A., Iannelli, F., Pozzi, G., and Pantosti, A. (2004). Tn2009, a Tn916-like element containing mef(E) in Streptococcus pneumoniae. Antimicrob. Agents Chemother. 48, 2037-2042. doi: 10.1128/AAC.48.6.20372042.2004

Gay, K., and Stephens, D. S. (2001). Structure and dissemination of a chromosomal insertion element encoding macrolide efflux in Streptococcus pneumoniae. J. Infect. Dis. 184, 56-65. doi: 10.1086/321001

Green, M. D., Beall, B., Marcon, M. J., Allen, C. H., Bradley, J. S., Dashefsky, B., et al. (2006). Multicentre surveillance of the prevalence and molecular epidemiology of macrolide resistance among pharyngeal isolates of group A streptococci in the USA. J. Antimicrob. Chemother. 57, 1240-1243. doi: 10.1093/jac/ dkl101

Hoskins, J., Alborn, W. E., Arnold, J., Blaszczak, L. C., Burgett, S., Dehoff, B. S., et al. (2001). Genome of the bacterium Streptococcus pneumoniae strain R6. J. Bacteriol. 183, 5709-5717. doi: 10.1128/JB.183.19.5709-5717. 2001

Iannelli, F., Pearce, B. J., and Pozzi, G. (1999). The type 2 capsule locus of Streptococcus pneumoniae. J. Bacteriol. 81, 2652-2654.

Iannelli, F., Santagati, M., Docquier, J. D., Cassone, M., Oggioni, M. R., Rossolini, G. M., et al. (2004). "Type M resistance to macrolides in streptococci is not due to the mef(A) Gene, but to mat(A) encoding an ATP-dependent efflux pump," in Proceedings of the Forty fourth Interscience Conference on Antimicrobial Agents and Chemoterapy, Washington, DC.

Iannelli, F., and Pozzi, G. (2004). Method for introducing specific and unmarked mutations into the chromosome of Streptococcus pneumoniae. Mol. Biotechnol. 26, 81-86. doi: 10.1385/MB:26:1:81

Iannelli, F., and Pozzi, G. (2007). "Protocol for conjugal transfer of genetic elements in Streptococcus pneumoniae," in Molecular Biology of Streptococci, eds R. Hakenbeck and G. S. Chhatwal (Norfolk: Horizon Scientific Press), 525-529.

Iannelli, F., Santagati, M., Santoro, F., Oggioni, M. R., Stefani, S., and Pozzi, G. (2014a). Nucleotide sequence of conjugative prophage Phil207.3 (formerly Tn1207.3) carrying the mef(A)/msr(D) genes for efflux resistance to macrolides in Streptococcus pyogenes. Front. Microbiol. 5:687. doi: 10.3389/fmicb.2014. 00687

Iannelli, F., Santoro, F., Oggioni, M. R., and Pozzi, G. (2014b). Nucleotide sequence analysis of integrative conjugative element Tn5253 of Streptococcus pneumoniae. Antimicrob. Agents Chemother. 58, 1235-1239. doi: 10.1128/AAC. 01764-13

Kelley, L. A., Mezulis, S., Yates, C. M., Wass, M. N., and Sternberg, M. J. (2015). The phyre 2 web portal for protein modeling, prediction and analysis. Nat. Protoc. 10, 845-858. doi: 10.1038/nprot.2015.053
Klaassen, C. H., and Mouton, J. W. (2005). Molecular detection of the macrolide efflux gene: to discriminate or not to discriminate between mef(A) and mef(E). Antimicrob. Agents Chemother. 49, 1271-1278. doi: 10.1128/AAC.49.4.12711278.2005

Lanie, J. A., Ng, W. L., Kazmierczak, K. M., Andrzejewski, T. M., Davidsen, T. M., Wayne, K. J., et al. (2007). Genome sequence of avery's virulent serotype 2 strain D39 of Streptococcus pneumoniae and comparison with that of unencapsulated laboratory strain R6. J. Bacteriol. 189, 38-51. doi: 10.1128/JB.01148-06

Leclercq, R., and Courvalin, P. (1991). Bacterial resistance to macrolides, lincosamide, and streptogramin antibiotics by target modification. Antimicrob. Agents Chemother. 35, 1267-1272. doi: 10.1128/AAC.35.7.1267

Lenart, J., Vimberg, V., Vesela, L., Janata, J., and Balikova Novotna, G. (2015). Detailed mutational analysis of vga(A) interdomain linker: implication for antibiotic resistance specificity and mechanism. Antimicrob. Agents Chemother. 59, 1360-1364. doi: 10.1128/AAC.04468-14

Martin, B., Prudhomme, M., Alloing, G., Granadel, C., and Claverys, J. P. (2000). Cross-regulation of competence pheromone production and export in the early control of transformation in Streptococcus pneumoniae. Mol. Microbiol. 38, 867-878. doi: 10.1046/j.1365-2958.2000.02187.x

Pearce, B. J., Iannelli, F., and Pozzi, G. (2002). Construction of new unencapsulated (rough) strains of Streptococcus pneumoniae. Res. Microbiol. 153, 243-247. doi: 10.1016/S0923-2508(02)01312-8

Pozzi, G., Iannelli, F., Oggioni, M. R., Santagati, M., and Stefani, S. (2004). Genetic elements carrying macrolide efflux genes in streptococci. Curr. Drug Targets Infect. Disord. 4, 203-206. doi: 10.2174/1568005043340641

Reinert, R. R., Ringelstein, A., van der Linden, M., Cil, M. Y., Al-Lahham, A., and Schmitz, F. J. (2006). Molecular epidemiology of macrolide-resistant Streptococcus pneumoniae isolates in Europe. J. Clin. Microbiol. 43, 1294-1300. doi: 10.1128/JCM.43.3.1294-1300.2005

Roberts, M. C., Sutcliffe, J., Courvalin, P., Jensen, L. B., Rood, J., and Seppala, H. (1999). Nomenclature for macrolide and macrolide -lincosamdestreptogramine B resistance determinants. Antimicrob. Agents Chemother. 43, 2823-2830.

Roberts, M. C. (2008). Update on macrolide-lincosamide-streptogramin, ketolide, and oxazolidinone resistance genes. FEMS Microbiol. Lett. 282, 147-159. doi: 10.1111/j.1574-6968.2008.01145.x

Rudolph, K., Bulkow, L., Bruce, M., Zulz, T., Reasonover, A., and Harker-Jones, M., et al. (2013). Molecular resistance mechanisms of macrolide-resistant invasive Streptococcus pneumoniae isolates from Alaska, 1986 to 2010. Antimicrob. Agents Chemother. 57, 5415-5422. doi: 10.1128/AAC.00319-13

Salles, C., Creancier, L., Claverys, J. P., and Mejean, V. (1992). The high level streptomycin resistance gene from Streptococcus pneumoniae is a homologue of the ribosomal protein S12 gene from Escherichia coli. Nucleic Acids Res. 20:6103. doi: 10.1093/nar/20.22.6103

Santagati, M., Iannelli, F., Cascone, C., Campanile, F., Oggioni, M. R., Stefani, S., et al. (2003). The novel conjugative transposon Tn1207.3 carries the macrolide efflux gene mef(A) in Streptococcus pyogenes. Microb. Drug Resist. 9, 243-247. doi: 10.1089/107662903322286445

Santagati, M., Iannelli, F., Oggioni, M. R., Stefani, S., and Pozzi, G. (2000). Characterization of a genetic element carrying the macrolide efflux gene mef(A) in Streptococcus pneumoniae. Antimicrob. Agents Chemother. 44, 2585-2587. doi: 10.1128/AAC.44.9.2585-2587.2000

Santagati, M., Lupo, A., Scillato, M., Di Martino, A., and Stefani, S. (2009). Conjugal mobilization of the mega element carrying $\operatorname{mef}(\mathrm{E})$ from Streptococcus salivarius to Streptococcus pneumoniae. FEMS Microbiol. Lett. 290, 79-84. doi: 10.1111/j. 1574-6968.2008.01408.x

Santoro, F., Oggioni, M. R., Pozzi, G., and Iannelli, F. (2010). Nucleotide sequence and functional analysis of the tet(M)-carrying conjugative transposon Tn5251 of Streptococcus pneumoniae. FEMS Microbiol. Lett. 308, 150-158. doi: 10.1111/ j.1574-6968.2010.02002.x

Scott, J. R., and Fischetti, V. A. (1983). Expression of Streptococcal M protein in Escherichia coli. Science 221, 758-760. doi: 10.1126/science.6192499

Seppala, H., Skurnik, M., Soini, H., Roberts, M. C., and Huovinen, P. (1998). A novel erythromycin resistance methylase gene (ermTR) in Streptococcus pyogenes. Antimicrob. Agents Chemother. 42, 257-262.

Sharkey, L. K., Edwards, T. A., and O’Neill, A. J. (2016). ABC-F Proteins mediate antibiotic resistance through ribosomal protection. mBio 7:e01975. doi: $10.1128 / \mathrm{mBio} .01975-15$ 
Smith, M. D., and Guild, W. R. (1979). A plasmid in Streptococcus pneumoniae. J. Bacteriol. 137, 735-739.

Sutcliffe, J., Tait-Kamradt, A., and Wondrack, L. (1996). Streptococcus pneumoniae and Streptococcus pyogenes resistant to macrolides but sensitive to clindamycin: a common resistance pattern mediated by an efflux system. Antimicrob. Agents Chemother. 40, 1817-1824.

Tait-Kamradt, A., Clancy, J., Cronan, M., Dib-Hajj, F., Wondrack, L., Yuan, W., et al. (1997). mefE is necessary for erythromycin-resistant $M$ phenotype in Streptococcus pneumoniae. Antimicrob. Agents Chemother. 41, 2251-2255.

Tatsuno, I., Isaka, M., Masuno, K., Hata, N., Matsumoto, M., and Hasegawa, T. (2018). Functional predominance of $\mathrm{msr}(\mathrm{D})$, which is more effective as mef(A)associated than $\mathrm{mef}(\mathrm{E})$-associated, over $\mathrm{mef}(\mathrm{A}) / \mathrm{mef}(\mathrm{E})$ in macrolide resistance in Streptococcus pyogenes. Microb. Drug Resist. doi: 10.1089/mdr.2017.0277 [Epub ahead of print].

Vitali, L. A., Di Luca, M. C., Prenna, M., and Petrelli, D. (2016). Correlation between genetic features of the $\operatorname{mef}(\mathrm{A})-\mathrm{msr}(\mathrm{D})$ locus and erythromycin resistance in Streptococcus pyogenes. Diagn. Microbiol. Infect. Dis. 84, 57-62. doi: 10.1016/j.diagmicrobio.2015.08.007
Weisblum, B. (1995). Erythromycin resistance by ribosome modification. Antimicrob. Agents Chemother. 39, 577-585. doi: 10.1128/AAC.39.3.577

Zhang, Y., Tatsuno, I., Okada, R., Hata, N., Matsumoto, M., Isaka, M., et al. (2016). Predominant role of $\mathrm{msr}(\mathrm{D})$ over $\operatorname{mef}(\mathrm{A})$ in macrolide resistance in Streptococcus pyogenes. Microbiology 162, 46-52. doi: 10.1099/mic.0. 000206

Conflict of Interest Statement: The authors declare that the research was conducted in the absence of any commercial or financial relationships that could be construed as a potential conflict of interest.

Copyright () 2018 Iannelli, Santoro, Santagati, Docquier, Lazzeri, Pastore, Cassone, Oggioni, Rossolini, Stefani and Pozzi. This is an open-access article distributed under the terms of the Creative Commons Attribution License (CC BY). The use, distribution or reproduction in other forums is permitted, provided the original author(s) and the copyright owner(s) are credited and that the original publication in this journal is cited, in accordance with accepted academic practice. No use, distribution or reproduction is permitted which does not comply with these terms. 